\title{
Self-Potential Inversion Using Genetic Algorithm
}

\author{
Maha Abdelazeem and Mohamed Gobashy* \\ National Research Institute of Astronomy and \\ Geophysics (NRIAG),Cairo, Egypt, and \\ * Department of Geophysics, Faculty of Earth Sciences, \\ King Abdulaziz University, Jeddah, Saudi Arabia \\ maazeem03@hotmail.com_*mgobashy@kau.edu.sa \\ Received: 13/12/2004 \\ Accepted: 26/2/2006
}

\begin{abstract}
A new technique is presented for automatic inversion of SP anomalies due to a polarized infinitely conductive simple geometrical source model structures, based on an artificial intelligence search strategy entitled genetic algorithm (GA) and the evolution theory. The genetic algorithm is used to find the minimum of fitness or cost function of the unknown depth, polarization angle, and shape factor. The study of synthetic examples shows fast and stable recovery of the true parameters even if the input data contain different percentages of noise. The GA leads to very realistic values for the inverted parameters in all tested examples and the root-mean squared error (rms) between the true and inverted field is accepted. The technique is further applied to real examples from Germany and Turkey.
\end{abstract}

\section{Introduction}

Self-potential (SP) prospecting is one of the oldest geoelectrical methods, and is still used in many fields of applied geophysics. The SP measurements refer to that part of the natural electrical field which is stationary in time, or nearly so, and whose current source system is generated and sustained by phenomena occurring underground within geologic structures. Many source mechanisms have been proposed to explain the genesis and time and space pattern of the SP field. The common aspect of the many source models is that an electric charge polarization is set up, which is responsible for electric current circulation in conductive rocks. For more details about this aspect, the reader is referred to 
Di Maio \& Patella (1991), Patella et al. (1997), Sailhac \& Marquis (2001) and Patella (2003). Hence the detected SP anomalies are simply the surface evidence of a more or less steady state of electric polarization. It follows that, in a final analysis, the SP inverse problem merely consists of finding the location and delineating geometry of any electric charge accumulation underground (Patella, 2003).

Self-potential data have been quantitatively interpreted using visual correlation between observed profiles, logarithmic curve matching, and characteristic points. In the last two decades, with the availability of fast computers, several numerical-based techniques have been developed to interpret SP anomalies. The use of available geometric source models, including charged points, lines, spheres, cylinders, and sheets provided very useful estimates of source parameters. These source parameters can simply be described by a self-potential (SP) anomaly expression produced by simple geologic forms that are represented as a continuous function of the parameter space.

The above mentioned techniques can be classified into three main categories as follows:

1. Graphical-based techniques. The different interpretation techniques available are using selected points on the anomaly curve. These were developed by Yüngül (1950); Paul (1965); Paul et al. (1965); Rao et al .(1970); Bhattacharya and Roy (1981) and Atchuta Rao \& Ram Babu (1983). The logarithmic curve matching were developed by Meiser (1962); and Murty and Haricharan, (1985). The drawback of the above methods is the complexity in case of numerous variable (El-Arabi, 2004).

2. Numerical-based techniques. These include analysis of SP fields using Fourier analysis in wavenumber domain (Asfahani et al., 2001), nomograms (Murty and Haricharan, 1985) and least-squares methods (Abdelrahman and Sharafeldin, 1997, and Abdelrahman et al., 1997b). Furthermore, derivative analysis and gradients have been also used (Abdelrahman et al., 1997a, 1998a, b, 2003, and Sundararajan et al., 1998). Although, the above techniques provide the best fit parameters using proper initial guess and characteristic points, they are greatly influenced by noise in the measured data and may lead to serious errors ( El-Arabi, 2004).

3. Complex analytical modeling-based techniques. More complex and advanced techniques based on physical tomography (Patella, 2003). Where imaging of a properly defined probability function of electric charge distribution in the surveyed volume was found to be consistent with the observed SP anomaly pattern.

In the first two categories, traditional algorithms have been successfully used. However, two main problems may limit the effectiveness of these techniques: 
first, by increasing the number of inverted parameters, the dimensionality of the energy surface increases, thereby greatly increasing the probability of the optimization stalling in a local minimum (Abdelrahman et al., 1998b). This is due to the fact that the SP inversion problem is an ill-conditioned and non-linear problem. Such ill-posed conditions were greatly damped where the depth and shape factor were estimated simultaneously by minimizing an objective function of depth and shape factor using the nonlinear simplex polytope algorithm (Gobashy, 2000). Secondly, discretization of the data and parameterization of model space, make the topology of the objective or fitness function exhibit several local minima (Ramillien and Mazzega,1999), as opposed to a continuous inverse where the number of solutions is infinite. Such conditions make the estimated models differ from the true and the appraisal problem arises. Moreover, ambiguity is inherited in the inversion of potential field data. Traditionally this problem has been tackled using detailed a priori information on the problem constraints. Such information is not commonly available in real geophysical problems. To detect the location of the global minima in the parameter space, classical techniques and even gradient-based methods that follow the steepestdescent direction are not a perfect choice. Unfortunately, even these search methods are local in scope and stop at the first local minimum they find (Ramillien, 2001).

This paper outlines the development of a scheme for the constraint inversion of SP anomalies through the use of evolutionary-based technique, the genetic algorithm (GA), which appears to be effective to overcome the above limitations. In the present approach, the shape factor, depth and polarization angle for the source models are estimated. Due to the evolutionarily structure of the GA, the appraisal problem is minimized and a good solution can be estimated. Synthetic and real examples are presented to test the validity of the proposed procedure.

\section{Problem Formulation}

The self-potential anomaly $V(x, h, \alpha, q)$ caused by a polarized infinitely conductive sphere or a cylinder in a homogeneous half-space (Fig. 1) at any point, $P\left(x_{j}\right)$ along the principal $x$-axis can be expressed (Yüngül, 1950; Bhattacharya and Roy, 1981; Murty and Haricharan, 1985; Abdelrahman et al., 1998a; ElArabi, 2004) as follows:

$$
V\left(x_{j}, h, \alpha, q\right)=M \frac{x_{j} \cos \alpha+h \sin \alpha}{\left(x_{j}^{2}=h^{2}\right)^{q}}
$$

where $M$ is the electric dipole moment, $h$ is the depth to the center of the sphere or cylinder, $x_{j}$ is a discrete point along $x$-axis where the observed anomaly is located, $q$ is the shape factor, and $\alpha$ is the polarization angle. The axis of the in- 


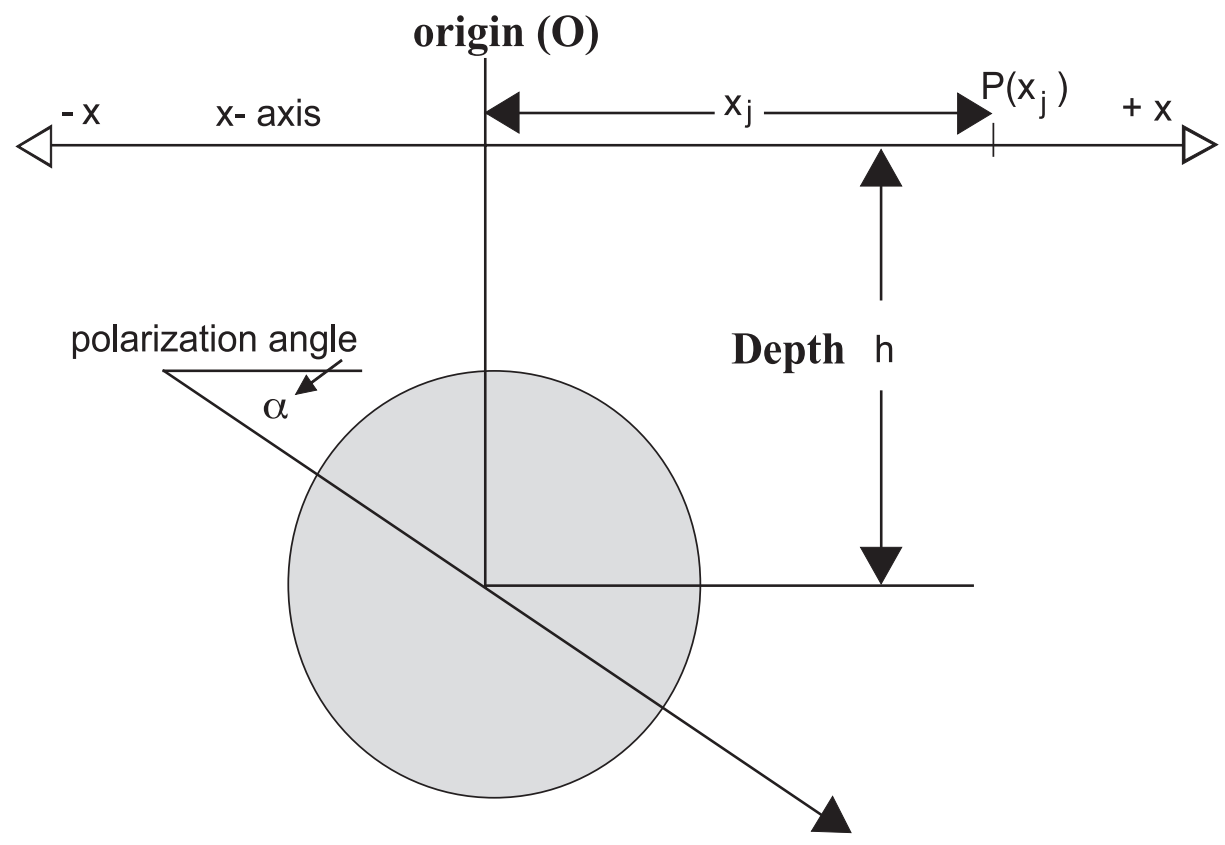

Fig. 1. Geometry of a 2-D cylinder or a sphere.

finite cylinder is perpendicular to the page and parallel to the Y-axis. In Eq. (1) $q=1$ for the horizontal cylinder, zero for a horizontal sheet and 1.5 for a sphere. $\alpha$ is measured from the surface to the polarization axis from the center of the sphere or cylinder to the surface (Fig. 1). The point $O$ is on the surface at a point vertically above the center of the anomaly source. The main target is to find, through a convenient numerical approach, the unknown parameters $h, \alpha$, and $q$ that best represent an observed anomaly $V_{o b s}$. The problem would be transformed into the problem of minimization of an objective or fitness function $\psi$ of the same unknowns. The form of this function, commonly used in geophysics, is simply:

$$
\psi\left(x_{j}, h, \alpha, q\right)=\sum_{i=1}^{m}\left|V_{o b s}\left(x_{j}, h, \alpha, q\right)-V_{\text {calc }}\left(x_{j}, h, \alpha, q\right)\right|
$$

Where $m$ is the number of data points and $V_{\text {calc }}$ is the calculated response. The target now is to minimize $\psi\left(x_{j}, h, \alpha, q\right)$ to estimate the unknowns under bound constraints on the unknown variables. In a mathematical form, this may be expressed as;

$$
\min _{\psi \subseteq R^{m}} \psi\left(x_{j}, h, \alpha, q\right)
$$


Such an optimization problem can be solved using an evolutionary scheme based on the genetic algorithm (GA) global optimizer. The next section gives a brief overview of the genetic approach and how to implement the SP problem.

\section{Principles of Genetic Algorithms}

Many optimization problems are very complex and quite hard to be solved by conventional optimization techniques. Over the last decade, genetic algorithms (GAs) have been extensively used as search and optimization tools in various problem domains (Wilson et al., 1994). The primary reasons for their success are their broad applicability, ease of use and global perspective (Goldberg, 1989; Billings et al., 1994; Boschetti et al., 1996; Stoffa and Sen, 1991 and Boschetti et al.,1997). Genetic algorithms mimic natural selection and biological evolution to achieve their power, and their operational characteristics are typically analogous to the evolution theory. Generally speaking, GAs initially works with a population of individuals, each representing a possible solution to a given problem. Each candidate solution, or individual, is generally represented as a string of bits (a set of binary character strings) analogous to chromosomes and genes in evolution theory. GAs assigns a fitness score to each individual based on the quality of the solution it represents, and highly fit individuals are reproduced by breeding with other individuals.

The fitness is a computation that evaluates the quality of the chromosome as a solution to a particular problem. By analogy with biology, the chromosome is referred to as the genotype, whereas the solution it represents is known as the phenotype. The translation process can be quite complicated. In timetabling and manufacturing scheduling GAs, for example, a chromosome is translated into a timetable or set of scheduled activities involving large numbers of interacting resources. The fitness computation will then go on to measure the success of this schedule in terms of various criteria and objectives such as completion time, resource utilization, cost minimization and so on. This complexity is reminiscent of biological evolution, where the chromosomes in a DNA molecule are a set of instructions for constructing the phenotypical organism. A complex series of chemical processes transforms a small collection of embryonic cells containing the DNA into a full-grown organism, which is then "evaluated" in terms of its success in responding to a range of environmental factors and influences.

New populations are continuously evolved over generations. The total number of solutions in one generation is called the population size denoted by $N_{\text {pop}}$. As different individuals compete for resources in the environment, those individuals that are fittest are more likely to survive and propagate their genetic material. Thus, during the evolution process, the quality of the population increases, leading to an optimal solution. Finally, the population is expected to 
converge towards an optimal solution to the encoded problem after a certain number of generations $\left(\mathrm{N}_{\text {gen }}\right)$ are completed (Hashem and Alex, 1999). A simple flowchart for GA is given in Fig. 2.

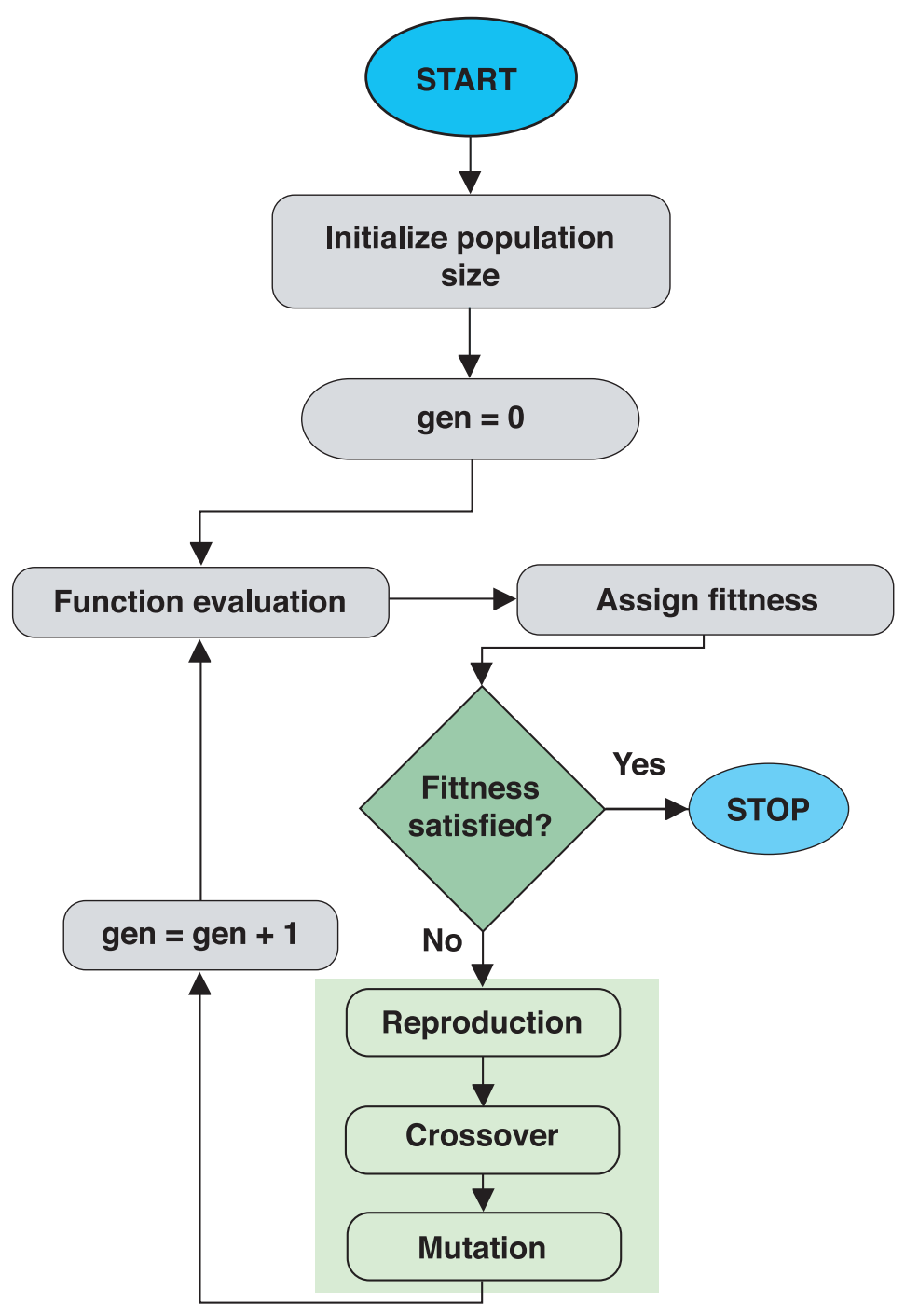

Fig. 2. A flow chart of the working principle of GA. 


\section{Selection of Initial Population}

As we previously mentioned, GA starts by a group of individuals or population, in order to avoid any bias at the beginning of the evolutionary operation, each of the parameters defining each individual in the initial population is initialized with uniformly distributed random number $R \in[0.0,1.0]$. The fitness is then calculated for each individual phenotype and stored in 1D array. The fitter the chromosome, the more times it is likely to be selected to reproduce.

In its simplest form, a genetic algorithm involves three types of reproduction operators: Selection, crossover and mutation, in the following sections, we discuss the reproduction operators used in this study.

\section{Reproduction Operators}

Reproduction operators of genetic algorithms provide a means to weed out bad solutions and to generate a new and better set of solutions during every iteration. Desirable characteristics of the parents are inherited by offspring during the reproduction phase by combining the best characteristics of both parents.

\section{Selection}

The process of selection of both parents in the study is carried out using a stochastic sampling mechanism that is mainly based on the Roulette wheel algorithm (Goldberg, 1989). The selection procedure is such that the probability of an individual being selected is proportional to that individual's fitness. However, directly using fitness as a measure of breeding probability suffers from a number of shortcomings (Goldberg, 1989 and Davis, 1991). In the present GA, the selection pressure or ranking procedure is implemented. In ranking theory, each individual is assigned a rank $r_{j}$ based on its fitness $S_{j}$. By convention, let $\mathrm{r}=$ 1 correspond to the fittest individual, and $\mathrm{r}=n p$ ( $n p:$ number of populations) to the least fit. Then the relative fitness $S^{\prime}$ in terms of the rank is:

$$
S_{j}^{\prime}=\frac{n p-r_{j}+1}{n p}, S_{j}^{\prime} \in[0,1]
$$

This relative fitness is then used as a measure of selection probability in the roulette wheel algorithm in place of the true fitness $S_{j}$. In this way, a constant fitness differential is imposed across the population.

\section{Crossover}

Crossover is the second reproduction process by which a portion of two fit parent individuals combine to produce two child individuals. There are three types of crossover operations typically used: single-point, two-point and uni- 
form crossover. Single-point crossover is usually performed by swapping the fragments between two parents at a random point along the bit-string. Crossover is generally applied to randomly paired strings with a moderate to high probability denoted $P \mathrm{c}$ (usually the value of $P \mathrm{c}$ falls between 60 and $100 \%$ ). The two-point crossover is used in this study (Fig. 3). It has the advantage of overcoming the end-point bias by selecting two randomly chosen splicing points along the string, and exchanging the string segments located in between these splicing points in a manner otherwise identical to one point crossover.

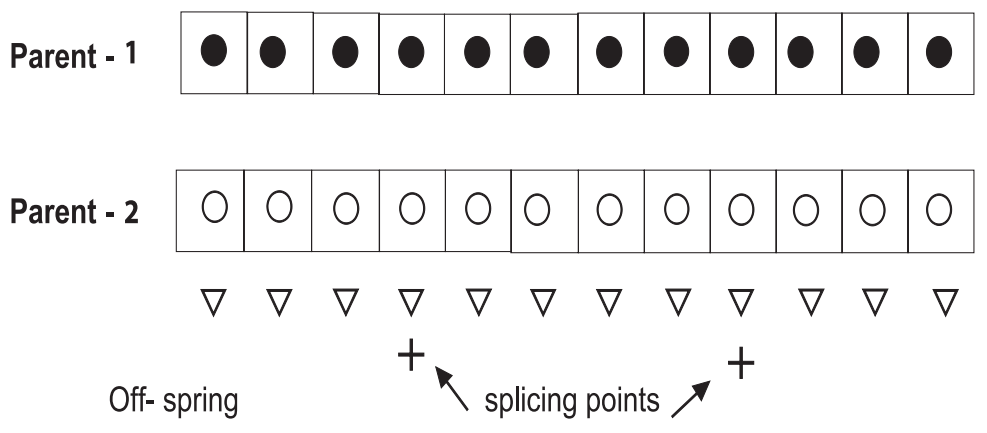

Child-1

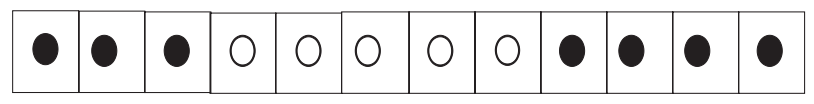

Child - 2

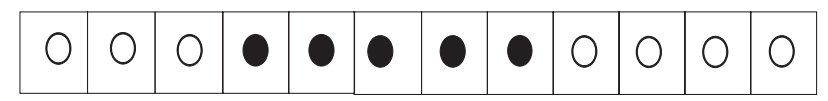

Fig. 3. Two-point crossover mechanism.

\section{Mutation}

Mutation is the third reproductive operator that provides a theoretical guarantee that no bit value is ever permanently fixed in all strings. Mutation introduces random modifications, thereby inducing a random walk through the search space. During mutation, with a low probability, a portion of the new individuals will be flipped to generate a new bit. In conjunction with the decimal/ decoding scheme, the uniform-one point mutation included in many GA routines is liable in getting stuck at Hamming Walls problem (Charbonneau, 2002). These problems arise, when a population of relatively good trial solution is being refined primarily by the action of mutation. The process of creep mutation used in these work overcome such a problem in a simple way. Once the digit has been targeted for mutation, the corresponding digit is either incremented or decremented with equal probabilities. 


\section{Adjustment of Mutation Rate}

In the present work, creep-mutation probability $\mathrm{p}_{\mathrm{m}}$ is self-adapted at the end of each generational iteration. The degree of clustering of the population is computed using as a measure, the normalized fitness difference between the best and median individuals (according to the fitness-based rank): $\Delta f=\left(f^{\max }\right.$ $\left.f^{\text {med }}\right) /\left(f^{\max }-f^{\text {med }}\right)$, where $f^{\max } \equiv f\left(x^{\max }\right)$, and $f^{\text {med }} \equiv f\left(x^{\text {med }}\right)$, and $\mathrm{x}_{\max }$, $\mathrm{x}_{\text {med }}$ are the parameter sets defining the best and median individuals, respectively. When $\Delta f \rightarrow 0$, the population is strongly clustered, while if $\Delta f \rightarrow 1$ the population is scattered across parameter space. The idea is to increase the probability $p_{m}$ in the former case, and decrease it in the later. The population clustering in this study is measured using the metric distance between the best and median as follows.

$$
\Delta_{d}=\frac{1}{n}\left(\sum_{j=1}^{n}\left(x^{\max }-x^{m e d}\right)^{2}\right)^{1 / 2}
$$

where, $n$ is the number of parameters in a solution vector $x$ (the three parameters; shape factor, depth and polarization angle). The mutation rate is varied according to:

$$
p_{m} \rightarrow\left\{\begin{array}{ll}
p_{m} \times \delta & \text { if } \Delta \leq \Delta^{\text {low }} \\
p_{m} & \text { if } \Delta^{\text {low }}<\Delta<\Delta^{\text {high }} \\
p_{m} \times \delta & \text { if } \Delta>=\Delta_{\text {low }}
\end{array}\right\}
$$

Where $\delta=1.5, \Delta^{l o w}=0.05, \Delta^{h i g h}=0.25$, and $\Delta$ stands for either $\Delta f$ or $\Delta_{d}$.

The above section gave a brief description to the GA used in this study, for more detail the reader can referee Charbonneau and Knapp, 1995, and Charbonneau, 2002.

\section{Application to Synthetic Examples}

\section{Synthetic Case 1}

We proposed a 2-D polarized infinitely conductive horizontal cylinder model with the shape factor $q=1.0$, the electric dipole moment $M=-300$, the depth to the center of the model $h=13$ units, and the polarization angle $\alpha=48$ degrees. We propose to measure the data along a profile extended along the surface from $x=-25$ to $x=+25$ units. The GA optimizer used has the following parameters (strategy parameters): number of individuals $=100$, number of generations $\left(N_{\text {gen }}\right)$ is 3500 , number of genes, i.e. number of significant digits retained in 
chromosomal encoding $=6$ (machine dependent), the crossover probability $=$ 0.85 which is the probability in which any one gene locus will mutate in any one generation, the mutation mode is taken variable, the initial mutation rate (probability $P_{m}$ ) is 0.005 . The bounds on the mutation rate are $=0.0005$ and 0.25 , respectively and the relative fitness differential $S_{j}$ is chosen as 1 .

Table 1. Results of inversion of synthetic example 1.

\begin{tabular}{|c|c|c|c|c|c|c|}
\hline & $\begin{array}{l}\text { Noise } \\
\text { added }\end{array}$ & $\begin{array}{c}\text { True } \\
\text { parameters }\end{array}$ & $\begin{array}{c}\text { Inverted } \\
\text { parameters }\end{array}$ & Error \% & $\begin{array}{l}\text { Optimum } \\
\text { fitness }\end{array}$ & $\begin{array}{l}\text { rms } \\
\text { error }\end{array}$ \\
\hline Shape factor $(q)$ & \multirow{3}{*}{$0 \%$} & 1.0 & 1.000 & 0 & \multirow{3}{*}{1.0059} & \multirow{3}{*}{0.0026} \\
\hline Depth $(h)$ & & 13 & 13.1546 & 1.18 & & \\
\hline Polarization $(\alpha)$ & & 48 & 48.0001 & 0 & & \\
\hline Shape factor $(q)$ & \multirow{3}{*}{$5 \%$} & 1.0 & 1.0002 & 0 & \multirow{3}{*}{0.0706} & \multirow{3}{*}{0.0299} \\
\hline Depth $(h)$ & & 13 & 13.202 & 1.55 & & \\
\hline Polarization $(\alpha)$ & & 48 & 47.862 & 0.28 & & \\
\hline Shape factor $(q)$ & \multirow{3}{*}{$13 \%$} & 1.0 & 1.0009 & 0 & \multirow{3}{*}{0.0277} & \multirow{3}{*}{0.067} \\
\hline Depth $(h)$ & & 13 & 13.225 & 1.73 & & \\
\hline Polarization $(\alpha)$ & & 48 & 47.432 & 1.18 & & \\
\hline
\end{tabular}

The results of the inversion using the GA approach are shown in Fig. (4a) and Table (1), which represents noise-free data. The root-mean square error (rms) is calculated and found to be $0.0026 \mathrm{mV}$. This is a measure of how close the calculated model response is to the measured data. The optimum fitness, $1.005 \mathrm{mV}$, and both the shape factor and the polarization angle are obtained with a high resolution ( $0 \%$ error). The depth is obtained with $1.18 \%$ relative error. The same example is inverted after adding 5\% and $13 \%$ noise to the synthetic data (Fig. 4b,c). Results are shown in Table (1). The table specifies the parameters, both true and inverted ones. It also shows the percentage of noise added to the synthetic data and the percentage of error in the parameters estimated. Similarly, relative errors in all parameters are calculated. The most resolved parameter is the shape factor $q$. The magnitude of the overall (rms) error is always less than $0.02 \mathrm{mV}$. The value of the minimum objective function is also given for each inversion process.

\section{Synthetic Case 2}

This is similar to synthetic case 1 . We proposed a polarized infinitely conductive spherical model with depth to center $h=10.5$ units, shape factor $q=1.5$, 

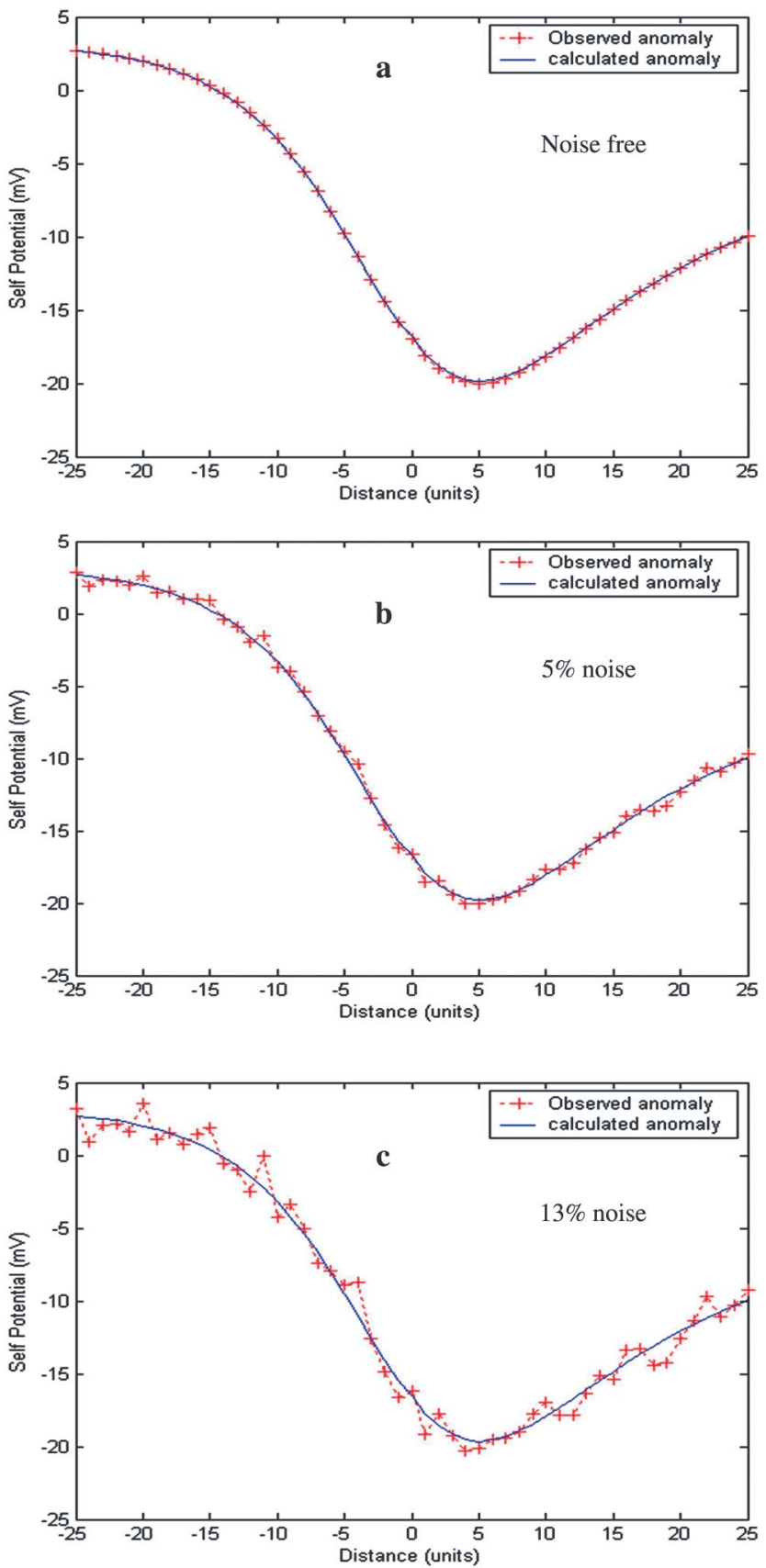

Fig. 4. The results of the inversion of a synthetic SP anomaly due to a 2-D horizontal cylinder model $(h=13, q=1.0$, and $\alpha=48)$ with $0 \%$ noise (a). Same data with $5 \%$ noise (b), and $13 \%$ noise (c). 
and polarization angle $\alpha=35$ degrees. For the sake of comparison, the GA strategy parameters are the same as those used in case 1 (number of individuals $=100, N_{\text {gen }}$ is 3500 , number of genes is 6 , the crossover probability $=0.85$, mutation probability $P_{m}$ is 0.005 . The bounds on the mutation rate are $=$ 0.0005 and 0.25 , and the relative fitness differential $S_{j}$ is chosen also as 1). Noise-free anomaly was firstly used and inverted using GA procedure. Results are shown in Fig. 5a and Table (2). The most resolved parameters after the inversion process is the shape factor $q$. The over all (rms) error is approximately zero. The optimum fitness is $0.0696 \mathrm{mV}$. The same inversion process is repeated with noise levels $6 \%$ and $14 \%$ (Fig. 5 b,c). The resolution of the different inverted parameters varies. The optimum fitness in the low noise is $0.56 \mathrm{mV}$, while in the high noise $0.26 \mathrm{mV}$. The (rms) error is less than $0.0044 \mathrm{mV}$ in both cases.

\section{Field Examples}

\section{Field Example 1}

The present approach is further applied to a field example from Germany, the graphite deposits in Bavarian Woods (Meiser, 1962). These deposits are situated in a hercynic gneissic complex. Conformably intercalated between paragneiss and crystalline limestone of the same age, they form seams that are to be designated as bituminous sediments of presumably Precambrian age. The target deposit lying between limestone and gneisses forming a parallel-running sequence of lenses, which are very variable in their thickness, this deposit can be approximated in some localities by simple geometrical models. The GA optimizer has been applied with a number of individuals $=100$ and number of genes equals to 3500 . All other GA strategy parameters are kept fixed as stated in the synthetic examples. The results of the inversion are shown in Fig. 6 and Table 3. The parameters obtained are: depth $h=35.50$ meters, polarization angle $\alpha=-62.99$ degrees, and shape factor $q=0.792$. The (rms) error calculated between the observed and calculated SP anomalies from the obtained parameters is $=1.7043 \mathrm{mV}$, which is much better than that of the earlier studies (Abdelrahman et al. 2003, $(\mathrm{rms}=25.3 \mathrm{mV}$ ). The obtained shape factor also suggests that a 2D horizontal cylinder model buried at a depth of 35.5 meters can represent the shape of the source body. These results (shape factor and polarization) are relatively close to those proposed by Meiser (1962) using double logarithmic net method and master curves, and Abdelrahman et al. (2003) using higher derivatives and least-squares method. Table (3) summarizes the above results. The calculated depth shows diversity from the results of Meiser and Abdelrahman (about $14 \mathrm{~m}$ ). We believe that our solution is close to reality because our data fitting is much better $(\mathrm{rms}=1.704)$. Moreover, synthetic study on the 

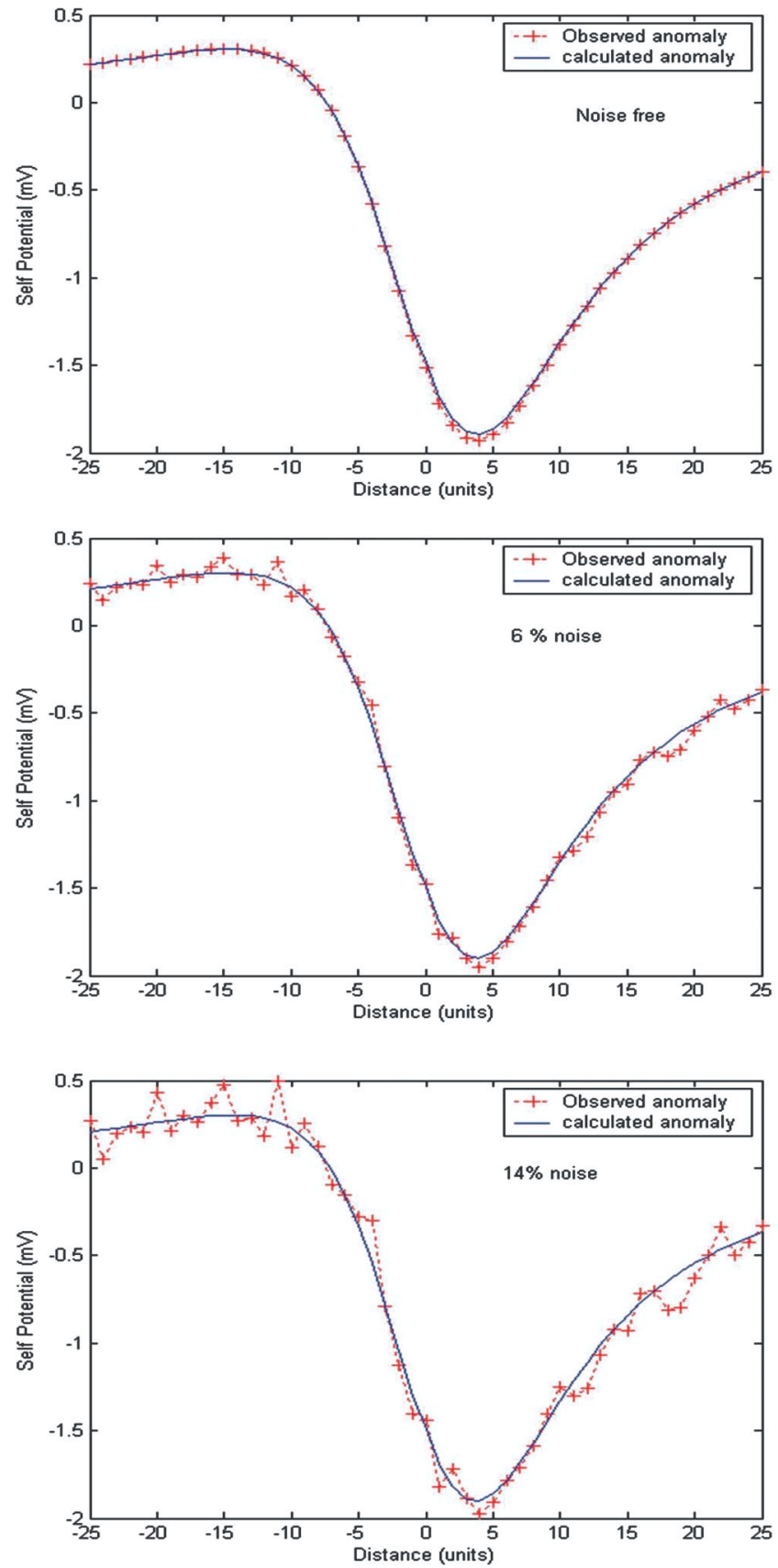

Fig. 5. The results of the inversion of a synthetic SP anomaly due to a spherical model $(h=$ $10.5, q=1.5$, and $\alpha=35$ ). The noise levels added to the data are $0 \%(a), 6 \%$ (b), and $14 \%$ (c). 
Table 2. Results of inversion of synthetic example 2.

\begin{tabular}{|c|c|c|c|c|c|c|}
\hline & $\begin{array}{l}\text { Noise } \\
\text { added }\end{array}$ & $\begin{array}{c}\text { True } \\
\text { parameters }\end{array}$ & $\begin{array}{c}\text { Inverted } \\
\text { parameters }\end{array}$ & Error \% & $\begin{array}{c}\text { Optimum } \\
\text { fitness }\end{array}$ & $\begin{array}{l}\text { rms } \\
\text { error }\end{array}$ \\
\hline Shape factor $(q)$ & \multirow{3}{*}{$0 \%$} & 1.5 & 1.4999 & 0.006 & \multirow{3}{*}{0.0698} & \multirow{3}{*}{0.0000433} \\
\hline Depth $(h)$ & & 10.5 & 10.6861 & 1.77 & & \\
\hline Polarization (a) & & 35 & 34.8000 & 0.571 & & \\
\hline Shape factor $(q)$ & \multirow{3}{*}{$6 \%$} & 1.5 & 1.50588 & 0.392 & \multirow{3}{*}{0.5613} & \multirow{3}{*}{0.0044} \\
\hline Depth $(h)$ & & 10.5 & 10.5289 & 0.275 & & \\
\hline Polarization (a) & & 35 & 34.84921 & 0.430 & & \\
\hline Shape factor $(q)$ & \multirow{3}{*}{$14 \%$} & 1.5 & 1.5120 & 0.8 & \multirow{3}{*}{0.2651} & \multirow{3}{*}{0.0092} \\
\hline Depth $(h)$ & & 10.5 & 10.36351 & 1.299 & & \\
\hline Polarization (a) & & 35 & 34.7874 & 0.607 & & \\
\hline
\end{tabular}

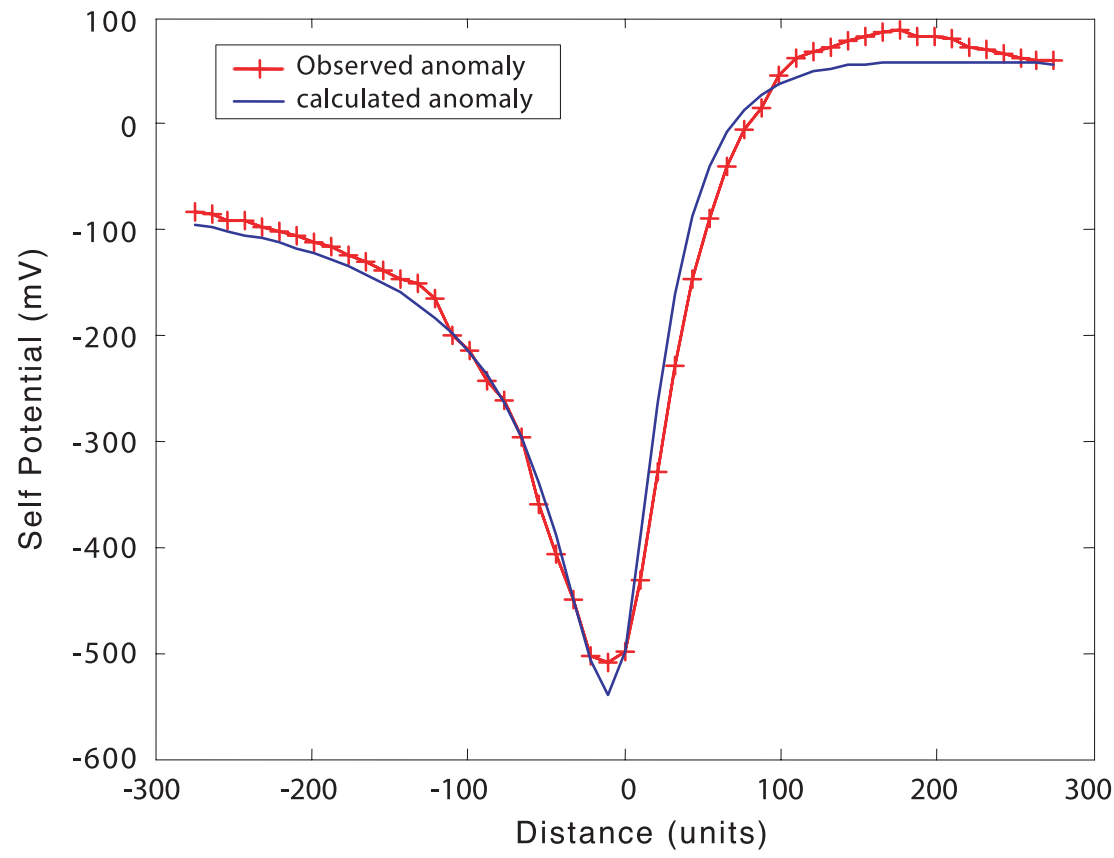

Fig. 6. Measured and calculated SP anomaly over a graphite ore body, southern Bavarian woods, Germany (after Meiser, 1962). With calculated $r m s=1.704 \mathrm{mV}$ at one unit $=$ 1 meter. 
Table 3. Results of the present technique using genetic algorithm applied to the field example and a comparison with the previous techniques.

\begin{tabular}{|c|c|c|c|c|}
\hline Parameter & Meiser (1962) & $\begin{array}{c}\text { Abdelrahman } \\
\text { et al., (2003) } \\
\text { (higher derivatives) }\end{array}$ & $\begin{array}{c}\text { Abdelrahman } \\
\text { et al., (2003) } \\
\text { (least squares) }\end{array}$ & $\begin{array}{c}\text { Present } \\
\text { approach }\end{array}$ \\
\hline Depth $(h)$ & 53 & 53 & 49.3 & 35 \\
\hline Polarization angle $(\alpha)$ & - & - & -55.7 & -62.9 \\
\hline Shape factor $(q)$ & - & 0.9 & 0.91 & 0.792 \\
\hline $\begin{array}{c}\text { Root mean square } \\
\text { error }(\mathrm{rms})\end{array}$ & - & - & 25.3 & 1.704 \\
\hline
\end{tabular}

same model with much higher noise level (13\%), assume confidence level of $\pm 1.73 \mathrm{mV}$ for the depth parameter.

\section{Field Example 2}

The second real example is from eastern Turkey. The anomaly named Süleymanköy lies in the Ergani copper district, $65 \mathrm{~km}$, south east of Elazig (Bhattacharya and Roy, 1981). The field measurements were performed and described in Yüngül (1950). They represent a polarized copper ore body. The anomaly has been digitized to 32 data points of 4.8 meters spacing. The inverted parameters are given in Table (4) to be compared with previous work results. The measured and inverted fields are shown in Fig. 7.

Table 4. Results of the Süleymanköy SP anomaly inversion as compared to previous work.

\begin{tabular}{|c|c|c|c|c|c|}
\hline Parameter & $\begin{array}{c}\text { Yüngül } \\
\mathbf{1 9 5 0}\end{array}$ & $\begin{array}{c}\text { Bhattacharya } \\
\text { \& Roy, } \\
\mathbf{1 9 8 1}\end{array}$ & $\begin{array}{c}\text { Asfahani } \boldsymbol{e t} \\
\text { al., 2001 }\end{array}$ & $\begin{array}{c}\text { Gobashy } \\
\mathbf{( 2 0 0 0 )}\end{array}$ & $\begin{array}{c}\text { Present GA } \\
\text { technique }\end{array}$ \\
\hline Depth $(h)$ in meters & 38.8 & 40 & 27 & 33.6 & 29.999 \\
\hline Polarization $(\alpha)$ & 21 & 15 & 17.25 & 15 & 11.549 \\
\hline Shape factor $(q)$ & h. cylinder & h. cylinder & h. cylinder & 0.99 & 0.961 \\
\hline
\end{tabular}

\section{Summary and Conclusion}

A new optimization strategy based on the genetic algorithm "global optimizer" is used to determine the parameters of a buried simple geometrical source model structures from their SP anomalies is used. The method uses the evolutionary characteristics that mimic nature's evolutionary principles to derive its search towards an optimal solution. The present approach uses a population of solutions in each iteration instead of a single solution used in classical tech- 


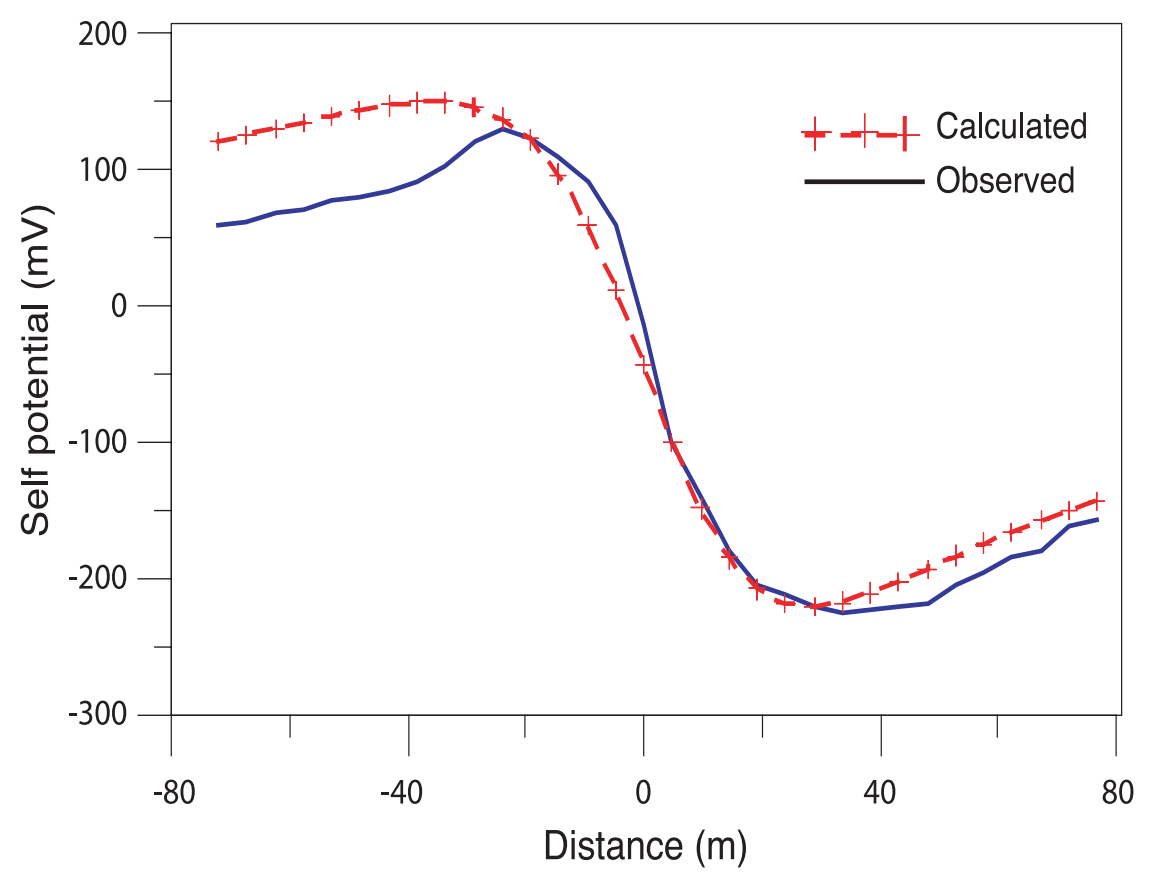

Fig. 7. Measured and calculated SP anomaly over the Süleymanköy SP anomaly, Turkey (after Yüngül, 1950) at one unit $=4.8$ meter.

niques. The outcome populations of solutions all converge to the optimum or minimum assuming a single global minimum exists. Moreover the present technique overcomes the ill-poseness and non-linearity of the SP problem. No further graphical processing or derivative analysis is used. The solution is obtained automatically for a residual SP field. Genetic algorithm is proven to providing reasonable results and proven to be effective in the interpretation of Sp data.

\section{References}

Abdelrahman, E.M., El-Araby, H.M., Hassaneen, A.G. and Hafez, M.A. (2003) New methods for shape and depth determinations from SP data, Geophysics, 68: 1202-1210.

Abdelrahman, E.M., Ammar, A.A., Hassanein, H.I., Hafez and M.A. (1998a) Derivative analysis of SP anomalies, Geophysics, 63: 890-897.

Abdelrahman, E.M., Hassaneen, A.Gh. and Hafez, M.A. (1998b) Interpretation of selfpotential anomalies over two-dimensional plates by gradient analysis, Pure and Applied Geophysics, 152: 773-780.

Abdelrahman, E.M. and Sharafeldin, M.S. (1997) A least-squares approach to Depth determination from self-potential anomalies caused by horizontal cylinders and spheres, Geophysics, 62: 44-48. 
Abdelrahman, E.M., Ammar, A.A., Sharafeldin, S.M. and Hassanein, H.I. (1997a) Shape and depth solutions from numerical horizontal self-potential Gradients, Journal of Applied Geophysics, 37: 31-43.

Abdelrahman, E.M., El-Araby, T.M., Ammar, A.A. and Hassanein, H.I. (1997b) A leastsquares approach to shape determination from residual self-potential Anomalies, Pure and Applied Geophysics, 150: 121-128.

Asfahani J., Tlas, M. and Hammadi, M. (2001) Fourier analysis for quantitative interpretation of Self-potential anomalies caused by horizontal cylinder and sphere, Journal of King Abdulaziz University, Earth Sciences, 13: 41-54.

Atchuta Rao, D. and Ram Babu, H.V. (1983) Quantitative interpretation of self potential anomalies due to two-dimensional sheet-like bodies, Geophysics, 48: 1659-1664.

Bhattacharya, B.B. and Roy, N. (1981) A note on the use of nomogram for self-potential anomalies, Geophys. Prosp., 29: 102-107.

Boschetti, F., Denitith, M.C. and List, R.D. (1996) Inversion of seismic refraction Data using Genetic Algorithms, Geophysics, 61: 1715-1727.

Boschetti, F, Mike, D. and List, R. (1997). Inversion of potential field data by Genetic algorithms, Geophysical Prospecting, 45: 461-478.

Billings, S., Kennett, B. and Sambridge, M. (1994) Hypocenter location: genetic algorithms incorporating problem specific information, Geophysical Journal International, 118: 693706.

Charbonneau, P. (2002) Release notes for PIKAIA 1.2, NCR/NT-451+STR, NCR Technical Note, Boulder, Colorado.

Charbonneau, P. and Knapp, B. (1995) A User's guide to PIKAIA 1.0, NCR Technical Note 418+LA ( Boulder: National center for Atmospheric Research).

Davis, L. (1991) Handbook of Genetic Algorithms, New York: Van Nostrand Reinhold.

Di Maio, R. and Patella, D. (1991) Basic theory of electrokinetic effects associated with earthquakes, Bollettino di Geofisica Tedorica ed Applicata, 33: 145-154.

El-Arabi, H. (2004) A new method for complete quantitative interpretation of self-potential anomalies, Journal of Applied Geophysics, 55: 211-224.

Gobashy M.M. (2000) Constraint inversion of residual self-potential anomalies, Delta Journal of Science, Tanta University, Egypt, 24.

Goldberg, D.E. (1989) Genetic Algorithms in Search Optimization and Machine Learning, Addison-Wesley, Reading, MA.

Hashem, T. and Alex, A.P. (1999) Using genetic algorithms to solve optimization problems in construction Engineering, Construction and Architectural Management, 6 (2): 121-132.

Meiser, P. (1962) A method of quantitative interpretation of self potential Measurements, Geophys. Prosp., 10: 203-218.

Murty, B.V.S. and Haricharan, P. (1985) Nomograms for complete interpretation of spontaneous polarization profiles over sheet-like and cylindrical two-dimensional sources, Geophysics, 50: 1127-1135.

Patella, D., Tramacere, A. and Di Maio, R. (1997) Modeling earth current Precursors in earthquake prediction, Annali d: Geofisica, $\mathbf{4 0}$.

Patella, D. (2003) Principles of electrography applied to self-potential electrokinetic sources and hydrogeological application, Water Resources Research, 39: 5SBH3-2-SBH3-15.

Paul, M.K. (1965) Direct interpretation of self-potential anomalies caused by Inclined sheets of infinite horizontal extensions, Geophysics, 30: 418-423.

Paul, M.K., Datta, S. and Banerjee, B. (1965) Interpretation of self potential anomalies due to localized causative bodies, Pure and Appl. Geophys., 61: 95-100. 
Ramillien, G. (2001) Genetic algorithms for geophysical parameter inversion from altimeter data, Geophys, J. Int., 147: 393-402.

Ramillien, G. and Mazzega, P. (1999) Non-linear altimetric geoid inversion for lithospheric elastic thickness and crustal density, Geophys, J. Int., 138: 667-678.

Rao B.S.R., Murthy, I.V.R. and Reddy, S.J. (1970) Interpretation of self-potential anomalies of some geometric bodies, Pure and Applied Geophysics, 78: 66-77.

Sailhac, P. and Marquis, G. (2001) Analytic potential for the forward and inverse modeling of SP anomalies caused by subsurface fluid flow, Geophys. Res. Lett., 28: 3.

Stoffa, P.L. and Sen, M.K. (1991) Nonlinear multiparameter optimization using genetic algorithms: Inversion of plane-wave seismograms, Geophysics, 56: 1794-1810.

Sundararajan, N., Rao, P.S. and Shunitha, V. (1998) An analytical method to interpret selfpotential anomalies caused by 2D inclined sheets, Geophysics, 63: 1551-1555.

Wilson, W.G., Laidlaw, W.G. and Vasudevan, K. (1994) Residual statics estimation using the genetic algorithm, Geophysics, 59: 766-774.

Yüngül, S. (1950) Interpretation of spontaneous polarization anomalies caused by spheroidal ore bodies, Geophysics, 15: 237-246. 


\section{حل المشكلة العكسية للجهد الذاتي باستخدام الخوارزم الجيني}

$$
\begin{aligned}
& \text { مها عبد العظيم محمد ، و محمد مصطفى غباشي }
\end{aligned}
$$

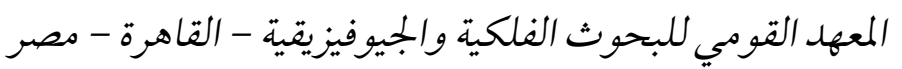

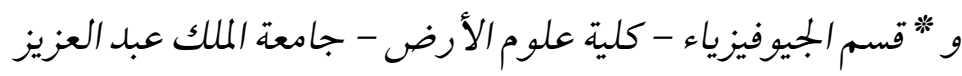

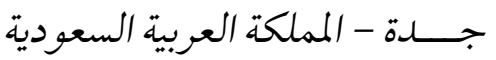

المستخلص. يهـدف هذا البحث إلى تقديم طريقة أوتوماتيكية جديدة

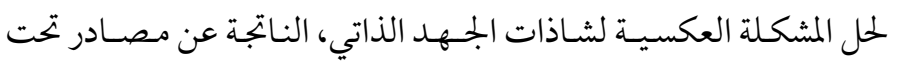

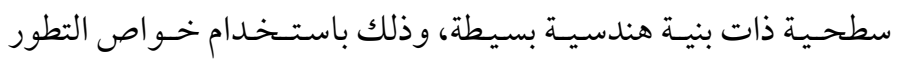

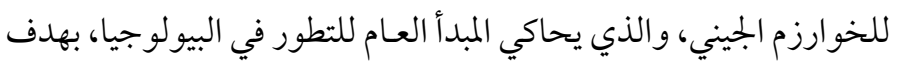

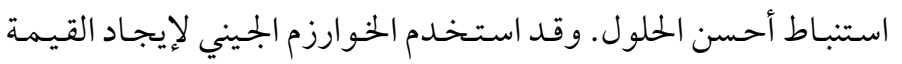

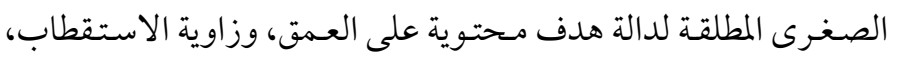

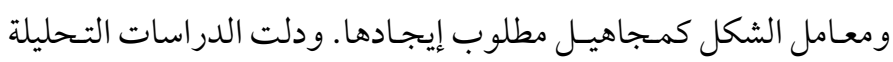

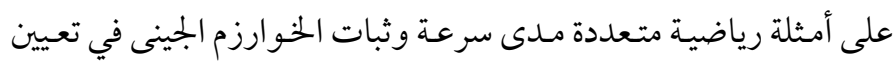

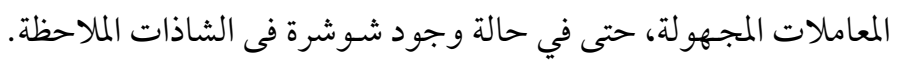

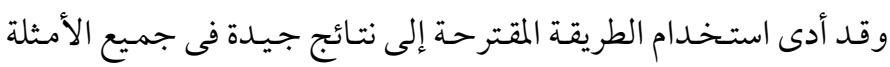

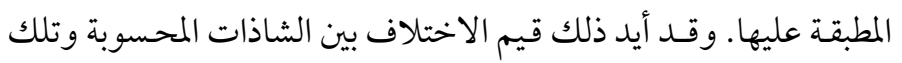

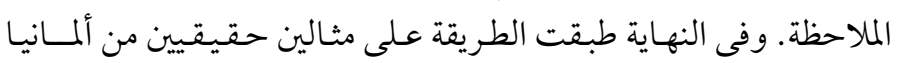

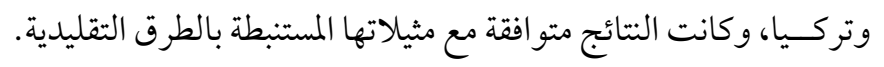

